\title{
A tai chi/qigong intervention for older adults living with HIV: a study protocol of an exploratory clinical trial
}

Gladys E. Ibañez ${ }^{1 *}$ D, Kristopher Fennie ${ }^{2}$, Linda Larkey ${ }^{3}, \mathrm{Nan} \mathrm{Hu}^{4,5}$, Angel B. Algarin ${ }^{1,6}$, Chelsea Valdivia ${ }^{4}$ and Helen Lavretsky ${ }^{7}$

\begin{abstract}
Background: Almost half of people living with HIV (PLWH) in the USA are over 50 years of age; this is expected to increase to $70 \%$ by 2020. Yet, few interventions exist for older PLWH that address psychological and physical symptoms combined, both prevalent in this population. There is a need to find innovative and accessible interventions that can help older PLWH to manage their symptoms. Mind-body interventions, like tai chi/qigong (TCQ), improve both physical and psychological health. TCQ is a series of slow, low-impact meditative movements that integrates breathwork, meditation, and stances.
\end{abstract}

Methods: The present study is an exploratory clinical trial that will evaluate the acceptability and feasibility of a 12week, small group TCQ intervention $(n=24)$, a sham qigong control condition $(n=24)$, and a standard of care control condition $(n=24)$ for older people living with HIV/AIDS. It will also explore any preliminary associations between the TCQ intervention and symptom alleviation. Participants will be recruited from community-based health and social services organizations in Miami, FL, and randomized to one of the 3 conditions.

Discussion: We will assess feasibility and acceptability through questionnaires and adherence to TCQ. We will assess preliminary associations with symptoms such as depression, anxiety, social support, chronic HIV-related fatigue, and clinical outcomes. These will be described through proportions, means, and changes over time through graphing techniques. Outcomes will be assessed at baseline, at post-intervention, and at 3 months follow-up. These preliminary analyses also will provide information necessary to estimate effect size and power needed for a larger clinical trial.

Trial registration: ClinicalTrials.gov NCT03840525. Registered on 16 July 2018.

Keywords: Qigong, Tai chi, HIV, Older adults, Intervention, Mind-body

\section{Introduction}

The prevalence of HIV in the USA is highest among older adults ( $45+$ years old), with people over 50 representing $45 \%$ of those who are living with HIV in the USA [1]. During 2012-2016, the largest percentage increase in prevalence rates $(55.7 \%)$ was among persons

\footnotetext{
* Correspondence: gibanez@fiu.edu

'Department of Epidemiology, Florida International University, 11200 SW 8th St AHC5, Miami, FL 33199, USA

Full list of author information is available at the end of the article
}

65 years and older [1]. Older PLWH also report more psychological and physical symptoms than their younger counterparts. A recent study found older PLWH reported more frequent agitation, depression, anxiety, apathy, irritability, and nighttime sleep disturbances than younger PLWH [2]. Older PLWH are 2-3 times more likely to suffer from depression than the general population $[3,4]$. Older PLWH also report lower levels of physical ability than younger PLWH [5]. HIV decreases bone mineral density and increases osteoporosis, making older 
PLWH at an increased risk of fall-related injuries $[6,7]$. Interventions are needed to address both the psychological and physical symptoms related to aging with HIV.

Mind-body interventions, like tai chi/qigong (TCQ), improve both physical and psychological health. TCQ is a slow, meditative movement exercise that uses breathwork, meditative focus, and fluid body movements to promote health. TCQ is considered "meditative movement" [8] and exercise for the mind as well as the body [9]. In a review of randomized clinical trials (RCTs) of TCQ and mental disorders, it was found that TCQ RCTs reported significant improvement in depression, stress, and anxiety, and had significant positive effects on mood and psychological well-being [10]. TCQ is known to also ameliorate physical health conditions such as cardiovascular conditions, hypertension, asthma, cancer [11-13], and diabetes [14]; all are common comorbidities found in PLWH. Moreover, this year, there have been several reviews and meta-analyses supporting the health benefits of TCQ including better immunological functioning [15], higher quality of life and less depression [16], anxiety [17], less hypertension [18], and general health promotion [19].

Despite growing evidence of the efficacy of TCQ to alleviate various chronic conditions, only a handful of intervention studies exist examining TCQ among PLWH. One RCT with PLWH found that TCQ was linked to quality of life and improved functional capacity [20], less emotion-focused coping [21], and lower HIVrelated psychological distress [22]. In sum, there is a gap in knowledge regarding the range of health benefits of TCQ for PLWH, as well as the acceptability and feasibility of a TCQ intervention with older PLWH. The purpose of this study is to describe the protocol for an exploratory clinical trial of a TCQ intervention with older PLWH. The primary outcome of the clinical trial is the acceptability and feasibility of a TCQ intervention, and the secondary outcomes are to explore the effects of TCQ on psychological and physical symptoms among older PLWH.

\section{Materials and methods}

\section{Overview}

The Gentle Empowering Movement (GEM) Study will measure the acceptability and feasibility of the TCQ intervention compared to a sham qigong (SQG) group, which is intended to control for physical activity and serve as an attention-control condition. There will also be a standard of care control group, which will not receive any services from the study. Both control groups will be offered the intervention after study completion.

This clinical trial was approved by the institutional review boards (IRB) at Florida International University and Arizona State University. It is funded by the National
Center for Complementary Integrative Health (NCCIH) and registered on www.clinicaltrials.gov [NCT03840525].

\section{Trial status}

The clinical trial enrolled its first participant on January 6 , 2020, and recruitment was ongoing until COVID-19. The study is currently suspended due to COVID-19 and will begin again once it is safe to reopen. The first cohort of intervention participants began February 7, 2020. This study protocol is version 5.0, created November 20, 2019. The anticipated completion date is December 2021.

\section{Study site}

The trial will be conducted at a partnering clinic, which is registered as a federally qualified health center focusing on minority populations in the inner city. The center's target population consists primarily of Hispanics 56\%, Haitians and African Americans 39\%, Whites, and others $5 \%$. The majority (86.9\%) earns an annual income at or below the federal poverty level.

\section{Theoretical framework for the intervention}

Researchers adapted Spirduso et al.'s exercise-cognition model [23] to fit the target outcomes and population (see Fig. 1). Our model focuses on the relationship between exercise (i.e., TCQ) and disease, using physical and psychological mediators. The theoretical framework links the components of TCQ to physical and psychological symptoms that in turn are expected to influence clinical outcomes. The secondary outcomes of the present clinical trial are these physical and psychological symptoms.

\section{Training instructors and DVD production}

The study co-investigator, a certified senior instructor of TCQ, trained intervention facilitators. The training lasted approximately $25 \mathrm{~h}: 8 \mathrm{~h}$ conducted via Zoom (v5.1.2; San Jose, CA) videoconferencing, followed by another $16-17 \mathrm{~h}$ of training in person in order to be certified. The trainer also trained the SQG control group facilitators. This training lasted $4-8 \mathrm{~h}$ including mock teaching. The senior TCQ instructor also met with the facilitators on a regular basis (weekly for the first month, then monthly) via phone and/or Zoom to discuss any issues that arise during the intervention. There is also an accompanying video that all facilitators and participants will have access to; participants use this video to help with their qigong home practice. This video was professionally produced by a video production company. A Spanish version of the video used for home practice sessions was also produced, and therefore, we have both English and Spanish versions of the TCQ and the SQG exercises for these study conditions, respectively, for participants to use during their home practice. These videos 


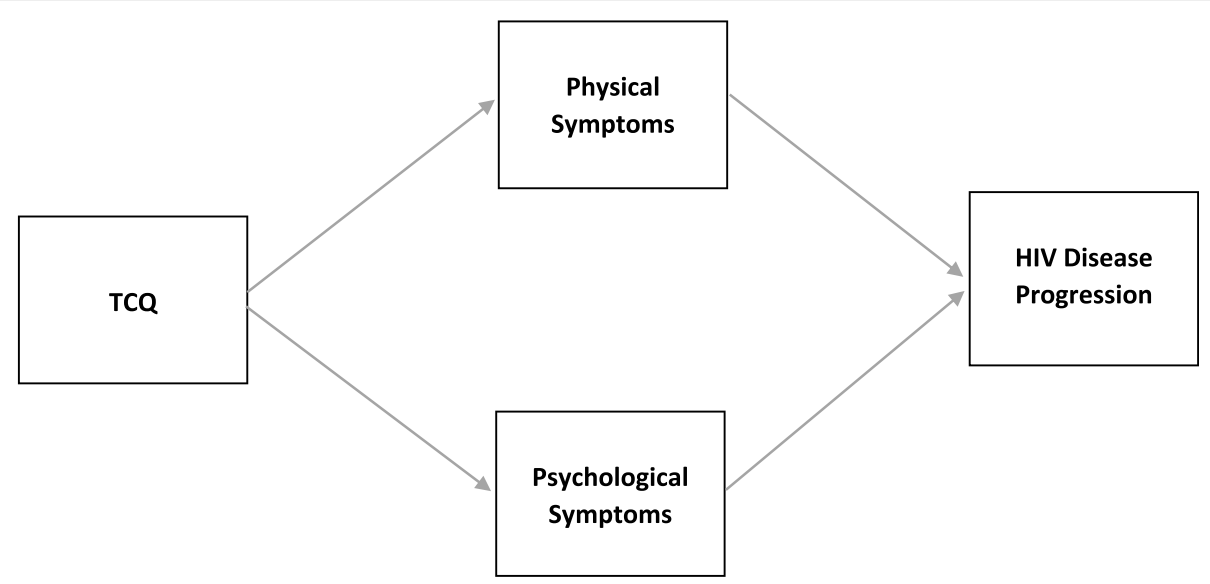

Fig. 1 Theoretical framework adapted from Spirduso et al. (2008) exercise-cognition model

were recorded using the TCQ and SQG facilitators. In addition to the video, each participant receives a booklet displaying all the movements learned in the group.

\section{Study design \\ Recruitment and screening}

This is a 3-arm randomized controlled exploratory clinical trial. Participants $(n=72)$ are recruited via clinic staff referrals, recruitment flyers, and/or clinic presentations. They are screened in person or over the phone by the research staff to confirm eligibility and scheduled for a baseline assessment. Participants are eligible if they are 50 years or older, living with HIV, able to provide consent, and willing and able to participate for the length of the intervention period (12 weeks). Participants are asked to provide the year in which they were first diagnosed with HIV by a healthcare professional, and the ability to provide consent will be assessed using the GAIN cognitive impairment scale, whereas the individual must score $\leq 10$ points to be deemed cognitively able [24]. Additionally, participants are provided with a thorough explanation of the study procedures and expected time commitment before they asked if they are willing and able to participate. Participants are excluded from the study if they report being unable to stand for 10-min segments (i.e., wheelchair or walker bound), or if the participant has substantial (i.e., regular weekly practice for more than 3 months in the past 12 months) experience with mind-body practices (tai chi, qigong, or yoga), they will be excluded because the control group may be contaminated by prior experience.

\section{The TCQ intervention}

The TCQ intervention is standardized and manualized and has a formal training program for instructors (www. instituteofintegralqigongandtaichi.org). The intervention content includes a series of repeated and easy to learn movements that are also the forms known to be linked to health benefits [25]. The TCQ intervention content includes three main components: (1) the three "intentful" corrections, (2) four movements based on the vitality method of qigong, and (3) six movements based on tai chi. The three "intentful" corrections form the basis for all subsequent movements and focuses on proper posture, breathwork, and clarity of mind as participants do the movements. Movements are taught standing and sitting on chairs for those who are unable to stand for of the entire class time (see Table 1 for list of movements). The steps and duration of the sham qigong group are in Table 2. The rationale of the sham is to serve as an attention-control group since the main research interest is in the meditative aspect of the qigong group. We attempt to ensure non-effectiveness of the sham qigong control group by the following: sham instructors are trained to keep the group as rote as possible, we conduct booster trainings as needed, and instructors are video

\begin{tabular}{|c|c|}
\hline \multirow[t]{2}{*}{ Week 1} & The three intentful corrections \\
\hline & Aligning, opening, and concluding tai chi easy sequences \\
\hline \multirow[t]{4}{*}{ Weeks 1-4 } & Vitality method_flowing motion \\
\hline & Vitality method_right and left bending of spine \\
\hline & Vitality method_front and back bending of spine \\
\hline & Vitality method_-reaching upward, stretching outward \\
\hline \multirow[t]{7}{*}{ Weeks 5-12 } & Spontaneous qigong \\
\hline & Channeling energy \\
\hline & Tai chi movement 1 - harmonizing yin and yang \\
\hline & Tai chi movement 2-brush knee send qi (chi) \\
\hline & Tai chi movement 3-cutting the path to clarity \\
\hline & Tai chi movement 4-watching clouds pass \\
\hline & Tai chi movement 5-gathering heaven and earth \\
\hline
\end{tabular}


Table 2 Main components of the SQG intervention

\begin{tabular}{ll}
\hline Weeks 1-12 Warm-up and cool down & Stretching neck \\
& Rolling shoulders \\
& Shrugging shoulders \\
& Body twist \\
& Hug your head \\
& Lunges \\
& Side stretch \\
& Reaching for the stars \\
& Dance step \\
& Swinging arms \\
& Hanging circles \\
& Overhead stretch \\
& Floor stretch \\
& Marching \\
Alternative movements & The swim \\
& Dusting the room \\
& The swing \\
& Reverse swim \\
\hline
\end{tabular}

recorded as they teach and intervention fidelity is assessed by a senior investigator using these videos.

\section{The sham qigong (SQG) and the standard of care control condition}

The SQG is a physical activity- and attention-control group that uses similar types and intensity of movements that are part of the TCQ intervention, but without the meditative state and breath focus that is present in the TCQ intervention. The third arm will be a standard of care control group and will receive no services. After trial completion, participants in both control conditions will be offered the intervention. See Table 3 for the characteristics of all 3 conditions.

\section{Criteria for discontinuing allocated interventions}

If a review of adverse events shows significant harms or if there is any breach of confidentiality, the study will be stopped, and a review of existing procedures will be conducted.

\section{Adherence/incentive strategies}

Based on some of the elements of social cognitive theory, we will use role-modeling, social support, and intrinsic reward to help participants adhere to the intervention. These strategies are the following: (1) the instructor will communicate with each participant when they come to the session to reinforce self-efficacy, (2) a monthly 1-page newsletter will be given to all participants with success stories and wellness information, and (3) a "buddy" system will be encouraged to promote participation. In addition, the research coordinator will make weekly phone calls as a reminder of upcoming sessions and to help promote the at-home practice. There will also be monetary incentives for participating in each of the assessments. Thirty dollars will be provided for the baseline and 2-week follow-up assessments, respectively, and $\$ 50$ for the 3-month follow-up assessment. However, if participants attend at least $80 \%$ of the intervention sessions, they will earn an additional $\$ 20$ at the 2-week follow-up assessment, that is, $\$ 50$. In addition, we will provide transportation assistance via UberHealth@ for those participants who need and request it.

\section{Sample size and randomization}

This is a pilot study that is not designed to test efficacy and as such is not powered for hypothesis testing. However, given a sample of 72 individuals, and an acceptability and feasibility proportion of $80 \%$, the precision of the measurement would be $95 \%$ confidence interval of $68-89 \%$, and by intervention group $61-92 \%$. We used a random block design to randomize participants into one of three treatment groups (TCQ, SQG, standard of care) in three equal blocks of 24 , whereby each block would have 24 participants randomized into the three treatment groups. The rationale for three blocks was logistical, so that assignment into the three treatment groups would be more even. The procedure for randomization involved creating a $\mathrm{SAS}^{\oplus}$ dataset that contained the structure necessary for the design, including an initial subject counter (1-72) and three treatment groups, nested in blocks. We then used the Plan Procedure in SAS using a seed to randomly assign each subject counter a treatment group within a block. The resulting list was imported into Excel, and a sequential participant identification was added. The randomization assignment list was housed with the study manager in a locked file cabinet. Neither the

Table 3 Descriptive characteristics of the TCQ intervention and control conditions

\begin{tabular}{|c|c|c|c|}
\hline & TCQ intervention & SQG control & Standard of care group \\
\hline Intervention features & $\begin{array}{l}\text { - Low-impact physical activity } \\
\text { - Focus on breath } \\
\text { - Meditative state } \\
\text { - Incidental social support }\end{array}$ & $\begin{array}{l}\text { - Low-impact physical activity with same/ } \\
\text { similar movements as TCQ intervention } \\
\text { - Incidental social support }\end{array}$ & - No exercise or activity program \\
\hline Dose/frequency & $\begin{array}{l}\text { - } 12 \text { weeks, } 1 / \text { week } 60 \text { min class } \\
\text { - } 2 \times \text { per week in first } 2 \text { weeks } \\
\text { - Approx. } 21 / 2 \mathrm{~h} \text { home practice/week }\end{array}$ & $\begin{array}{l}\cdot 12 \text { weeks, } 1 / \text { week } 60 \text { min class } \\
\cdot 2 \times \text { per week in first } 2 \text { weeks } \\
\text { - Approx. } 21 / 2 \mathrm{~h} \text { home practice/week }\end{array}$ & $\cdot n / a$ \\
\hline Controls for & - Unique focus; breath and meditative state & - Low-impact physical activity & $\cdot n / a$ \\
\hline
\end{tabular}


interviewer nor the statistician had access to the list. Immediately prior to or after an enrolled person's baseline interview, the study manager assigned the participant to the next available treatment condition, in sequential order (Fig. 2).

\section{Blinding}

For logistical reasons, the study will be partially blinded. Those randomized to the TCQ and SQG conditions will be blinded to which group they were assigned, whereas the standard of care control group will know they are assigned to the control condition based on format and content of the condition. Data collectors and analysts will be blinded to group assignment. Participants will also be blinded. Success of the blinding will be assessed by asking participants to guess which condition they attended during the 3-month follow-up interview. The study research coordinator and principal investigator are unblinded throughout the study.

\section{Assessments}

Once eligibility is determined, participants are scheduled for a baseline assessment. All assessments are conducted in a private research office space. Bilingual research staff will be trained to conduct the survey in order to collect the data, if needed. Participants are consented and administered the baseline measures using computer-assisted personal interviewing procedures with REDCap software. Baseline assessments last approximately $45 \mathrm{~min}$ and include demographic items, psychological and physical symptoms measures, adherence items, and viral load. At 2-week follow-up and 3month follow-up, the same measures are administered (see Table 4 for a description of all measures). Most measures have a Spanish version, but for those that do not, a backtranslation procedure was used to translate measures [37].

\section{Acceptability measures}

Acceptability is measured by administering a brief satisfaction survey to participants after each intervention session and comparing them to the control conditions. We collect acceptability metrics such as attendance, information on home practice sessions including how often they practiced and how long each practice session was, and satisfaction with the DVD and practice sessions. Acceptability of the home practice sessions and adherence strategies is collected during weekly calls to participants. Based on Sekhon et al.'s [38] work, there are specific benchmarks to determine the acceptability of the intervention (see Table 5).

\section{Feasibility measures}

Feasibility metrics include percentage of those who are eligible, consented, and randomized. The percentage of who completed the 2-week and 3-month follow-up assessments are calculated. The percentage of who did the home practice sessions are collected via weekly call. The intervention facilitators complete a survey at the end of

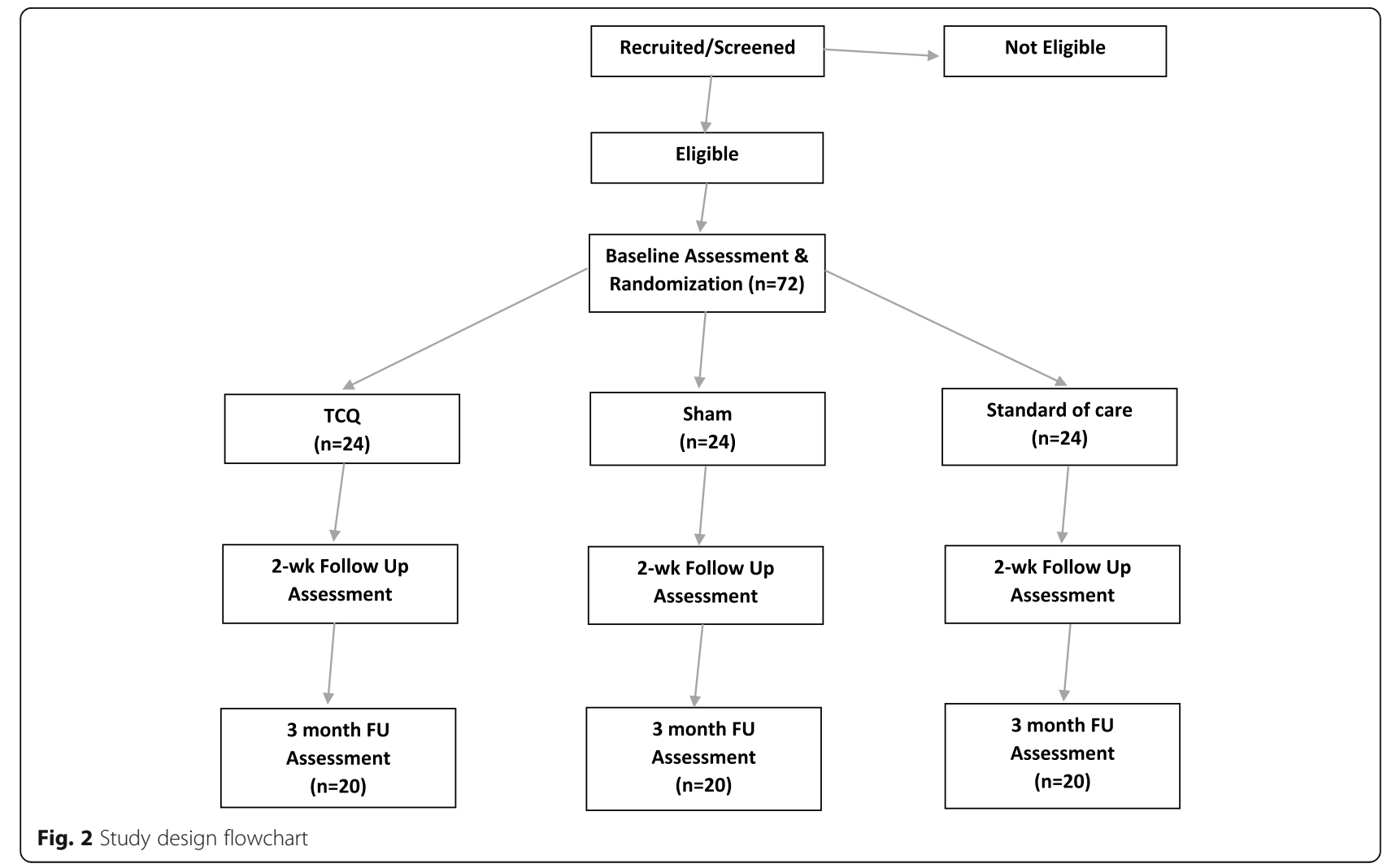


Table 4 All measures and time of administration

\begin{tabular}{|c|c|c|c|c|c|c|}
\hline Quantitative measures & $\begin{array}{l}\text { No. of items/ } \\
\text { psychometrics }\end{array}$ & Screen & $\mathrm{BL}$ & $\begin{array}{l}\text { Weekly/during } \\
\text { intervention }\end{array}$ & 2-week FU & 3-month FU \\
\hline \multicolumn{7}{|l|}{ Eligibility [24] } \\
\hline Age, HIV status (self-report/lab test) & & $x$ & & & & \\
\hline Any mind-body practice in past 12 months & & $x$ & & & & \\
\hline GAIN cognitive impairment & 6 items $/ \mathrm{a}=.87$ & $x$ & & & & \\
\hline Can stand up for $10 \mathrm{~min}$ & & $x$ & & & & \\
\hline Demographics and covariates [26-30] & & & $x$ & & & \\
\hline HIV-related stigma & 6 items $/ a=.75$ & & & & & \\
\hline Social support scale & 12 items $/ \mathrm{a}=.94$ & & & & & \\
\hline Drug use (DAST) & 20 items $/ \mathrm{a}=.92$ & & & & & \\
\hline Alcohol (AUDIT) & 10 items $/ \mathrm{a}=.83$ & & & & & \\
\hline \multicolumn{7}{|l|}{ Psychological symptoms $[31,32]$} \\
\hline CES-D scale & & & $x$ & & $x$ & $x$ \\
\hline Depression, Anxiety and Stress Scale (DASS-21) & 21 items $/ \mathrm{a}=.86$ & & & & & \\
\hline \multicolumn{7}{|l|}{ Physical symptoms $[33,34]$} \\
\hline The Revised Sign and Symptom Checklist for HIV & 45 items $/ a=.71-.91$ & & $x$ & & $x$ & $x$ \\
\hline HIV-related Fatigue Scale (MAF) & 16 items $/ a=0.88$ & & $x$ & & $x$ & $x$ \\
\hline \multicolumn{7}{|l|}{ Clinical [35] } \\
\hline 5-item medication adherence questions MMAS- 8 & 8 items $/ a=.75$ & & $x$ & & $x$ & $x$ \\
\hline Viral load (lab results) & & & $x$ & & $x$ & $x$ \\
\hline Feasibility & & & $x$ & $x$ & $x$ & \\
\hline Acceptability & & & & $x$ & $x$ & \\
\hline $\begin{array}{l}\text { Feasibility of implementation in clinic setting } \\
\text { (interventionists reporting) }\end{array}$ & & & & & $x$ & \\
\hline \multicolumn{7}{|l|}{ Intervention fidelity [36] } \\
\hline Meditative Movement Inventory & 9 items $/ a=.86-.90$ & & & $x$ & $x$ & \\
\hline Intervention Fidelity Checklist (for senior trainer) & & & & $x$ & & \\
\hline
\end{tabular}

each intervention cohort (12 weeks duration) to assess whether the intervention was feasible to implement in a clinic setting (see Table 5).

\section{Intervention fidelity}

All intervention and control group sessions are video recorded and stored on a secured server accessible only to the principal investigator and co-investigators. Our senior trainer views $10-20 \%$ of these video recordings and rates them on intervention fidelity using an intervention fidelity measure developed for the study.

\section{Confidentiality}

All data collected is identified only by a participant identification number to maintain confidentiality. All paperbased records are also kept in a locked file cabinet in the research office which was also locked nightly. In addition, data collected via laptops are encrypted and stored on a secured cloud server.

\section{Data analysis plan}

Data obtained through REDCap ${ }^{\bullet}$ will be imported into $\mathrm{SAS}^{\bullet}$ version 9.4. Acceptability and feasibility data will be collected through paper-based questionnaires and logs and tracked through $\operatorname{RedCAP}^{\circ}$ and imported into $\mathrm{SAS}^{\ominus}$. Paper-based questionnaires and $\operatorname{logs}$ will be reviewed by a research staff for completeness and consistency. Paper-based questionnaires will be double entered to ensure accuracy of data entry. Prior to analysis, data will be cleaned by examining frequencies, means, medians, and ranges to identify logical errors. Instruments will be coded according to their respective scoring instructions. Unless specified by the instrument, missing items will be imputed based on averaging values within the instrument for a given individual, if no more than $15 \%$ of items are missing. Otherwise, that instrument will be set to missing for the individual.

Because this is an exploratory clinical trial, analyses will be primarily descriptive; hypotheses will not be assessed through statistical significance, in accordance 
Table 5 Acceptability and feasibility definitions and benchmarks

\begin{tabular}{|c|c|c|}
\hline & Definition & Benchmark \\
\hline \multicolumn{3}{|c|}{ Aspects of acceptability being measured (adapted from Sekhon et al. [38]) } \\
\hline Burden & Discontinuation/non-attendance & $\begin{array}{l}<20 \% \text { of TCQ and the SQG condition will drop out at } 3 \text { months } \\
\text { follow-up. }\end{array}$ \\
\hline Ethical consequences & Any side effects with intervention & $\begin{array}{l}<10 \% \text { of participants will report any physical soreness or } \\
\text { difficulties in doing TCQ. }\end{array}$ \\
\hline \multirow[t]{2}{*}{ Experience } & \multirow[t]{2}{*}{ Participant's experience and satisfaction } & > 80\% TCQ participants will perceive intervention positively. \\
\hline & & $>80 \%$ TCQ participants will rate it very satisfactory or satisfactory. \\
\hline Affective attitudes & Participants attitudes toward the intervention & $\begin{array}{l}>80 \% \text { of participant in TCQ condition will report positive attitudes } \\
\text { about it (very good/good). }\end{array}$ \\
\hline \multirow[t]{2}{*}{ Opportunity costs } & \multirow[t]{2}{*}{ Adherence and participation } & $\begin{array}{l}\text { Participants in both TCQ and SQG conditions will attend at least } \\
75 \% \text { of all sessions. }\end{array}$ \\
\hline & & $\begin{array}{l}\text { Participants in the TCQ condition will adhere to home practice at } \\
\text { least } 75 \% \text { of the time expected (i.e., } 112 \text { out of } 150 \text { min/week). }\end{array}$ \\
\hline \multirow[t]{2}{*}{ Intentions } & \multirow[t]{2}{*}{ Willingness to participate } & $>80 \%$ TCQ participants would be willing to participate again. \\
\hline & & $>80 \%$ TCQ participants intend to continue qigong practice. \\
\hline \multicolumn{3}{|c|}{ Aspects of feasibility being measured (adapted from Bowen et al. [39]) } \\
\hline Demand & Likelihood that intervention will be used & $\begin{array}{l}>80 \% \text { of TCQ participants will report any TCQ practice in the } \\
\text { past week. }\end{array}$ \\
\hline \multirow[t]{3}{*}{ Implementation } & \multirow{3}{*}{$\begin{array}{l}\text { Can the intervention be implemented in a } \\
\text { setting, often a real-world setting }\end{array}$} & Trainers will execute intervention $>80 \%$ of the time. \\
\hline & & $\begin{array}{l}>80 \% \text { of trainers will rate resources needed to implement as } \\
\text { very good/good. }\end{array}$ \\
\hline & & $>80 \%$ of trainers will report positive effects on population. \\
\hline Practicality & $\begin{array}{l}\text { Extent to which an intervention can be delivered } \\
\text { given the limited resources }\end{array}$ & $\begin{array}{l}>80 \% \text { of trainers will report participant's ability to do the } \\
\text { intervention as very good/good. }\end{array}$ \\
\hline \multirow[t]{2}{*}{ Integration } & \multirow[t]{2}{*}{ Extent to which the intervention fits the system } & $\begin{array}{l}>80 \% \text { of trainers will perceive intervention fits the clinic } \\
\text { infrastructure as very good/good. }\end{array}$ \\
\hline & & $\begin{array}{l}>80 \% \text { of trainers perceive the sustainability of the intervention } \\
\text { as very good/good. }\end{array}$ \\
\hline
\end{tabular}

with recommendations for analysis of pilot studies [40]. Feasibility, acceptability, and clinical outcomes will be described through proportions, means, and changes over time, and $95 \%$ confidence intervals (CI) will be calculated to estimate precision. Regarding clinical outcomes, researchers will focus on estimating treatment effect sizes and respective 95\% CI, which will help to determine the sample size needed for a larger scale RCT. Baseline demographic and clinical characteristics, including psychological and physical symptoms, will be summarized by treatment condition and compared for even distribution of characteristics to assess effectiveness of the randomization.

To address acceptability among participants, data will be used from the satisfaction survey which will be administered weekly during the intervention and at postintervention. Individual items from the survey such as percent sessions attended, frequency of home practice sessions, average length of home practice sessions, and questions on opinion of the intervention sessions, home practice sessions, and video will be plotted over time to examine variability of acceptability measures over time.
Next, participant's average score over the intervention period will be averaged. These averages, as well as the items from the post-intervention satisfaction survey, will be categorized into acceptable or not acceptable. Benchmarks used to determine acceptability and feasibility were predetermined in collaboration with the sponsoring agency (see Table 5). From these data, we will determine the proportion of participants who rate the intervention as acceptable. To estimate precision, $95 \% \mathrm{CI}$ will be calculated using Wilson's score method, which uses asymptotic variance and is appropriate for small sample sizes [41]. The formula is as follows: $\left(2 n p+z^{2} \pm \sqrt{ }\left(z^{2}+4 n p q\right)\right) /$ $2\left(n+z^{2}\right)$. We also will conduct the above analysis on both control conditions. Certain questions on the satisfaction survey will differ with the control condition, but we anticipate similar levels of acceptability among the three conditions. Lastly, we plan to repeat these descriptive analyses among three separate strata: gender, current drug users, and age groups (50-65 and 65+), assuming variation within strata. We anticipate that there could be differences in acceptability among these different groups. 
Clinical, psychological, and physical outcomes will be assessed at baseline, at post-intervention, and at 3 months follow-up. The Depression, Anxiety and Stress Scale-21 (DASS-21) instrument will be used to assess depressive symptoms, anxiety-related symptoms, and stress. To assess physical symptoms, the Revised Sign and Symptom Checklist for HIV and the HIV-related Fatigue Scale will be used [33, 34]. To assess clinical outcomes, adherence to antiretroviral medicine will be measured using a 5-item medication adherence questionnaire. Adherence will be set at $95 \%$. Viral load at baseline and at 3 months (if available) will also be collected.

Gauging preliminary efficacy is helpful in determining effect sizes; thus, while we will not be testing for statistical significance, we will conduct the below described models and examine confidence intervals, in order to help estimate effect size. We will describe outcome data using univariate and bivariate statistics. For categorical outcomes, we will look at frequencies at each time point. For dichotomous outcomes, a repeated measures logistic regression will be completed using the Genmod and Glimmix Procedures that will include group, time, and a group by time interaction, followed by multiple comparisons of the interaction term to determine changes over time within each time condition as well as differences among the groups at post-intervention and 3-month follow-up. A 95\% CI will be used to help determine effect size. Univariate analyses will be conducted for continuous outcomes, including mean, median, range, and standard deviation. Distributions will be assessed for normality. Univariate analyses will be conducted on the outcomes stratified by gender, current drug use, and age groups. Then, a repeated measures linear regression will be conducted, using the Mixed Procedure, with a group, time, and group by time interaction, followed by multiple comparisons. The Power Procedure in $\mathrm{SAS}^{\circ}$ and GPower ${ }^{\bullet}$ will be used to estimate the sample size needed to test differences in outcomes among the three treatment conditions in a future RCT.

\section{Interim analyses}

In addition, interim analyses will be conducted. A data safety monitoring board (DSMB) was established for this clinical trial. It will meet every 6 months to review the progress of the clinical trial, and determine if discontinuation or continuation is justified based on the interim report. The interim analyses will include information on accrual, baseline characteristics, and other general information on study status. This report will also contain data on study outcomes, safety, and serious adverse events (AEs).

\section{Oversight and monitoring}

This is a feasibility study being conducted at one site, and therefore, there is no coordinating center. However, the data management team, consisting of the principal investigator, co-investigators, consultant, and research coordinator, meet monthly to discuss study progress. In addition, the principal investigator also meets with staff on a weekly basis to review study progress.

The DSMB consists of at least 3 members. Two members constitute a quorum. The members have been approved by the $\mathrm{NCCIH}$ and will include at least one member with human subject research monitoring expertise, at least one member with relevant disease expertise (such as an M.D. or equivalent), at least one member with expertise in the intervention or observation technique under study, and a Ph.D.-level biostatistician. The DSMB responsibilities include the following: review the research protocol, study materials, and all proposed revisions; evaluate the progress of the study; consider factors that may impact the safety of the study participants; and make recommendations to the $\mathrm{NCCIH}$, the Grantee Institution, the principal investigator (PI), and the IRB concerning continuation, termination, or other modifications of the study. Members of the DSMB have no financial, scientific, or other conflict of interest with the study. Current or past (within 3 years) collaborators, including any individual involved in the design, conduct, or analysis of the study; associates; and direct reports of the PI are not eligible to serve on the IMC. Meetings are attended, when appropriate, by the study principal investigators $(\mathrm{PI}(\mathrm{s}))$ and members of the study team. A formal report containing the recommendations for continuation or modifications of the study, prepared by the DSMB, will be sent to the study PI(s). It is the responsibility of the study $\mathrm{PI}(\mathrm{s})$ to distribute this report to all co-investigators and the $\mathrm{NCCIH}$ and to the IRB associated with the study. Each DSMB report should conclude with a recommendation to continue or to terminate the study.

All research staff will also be trained on the definitions of SAEs and AEs and its reporting procedures. Staff will notify the PI immediately after the occurrence of an SAE or AE. SAEs will be reported to NIH and to the IRB within $24 \mathrm{~h}$, with a written report within $48 \mathrm{~h}$. All AEs that occur during the study will be documented and reported by the investigators to NIH and the FIU IRB at the time of their continuing annual reviews. The principal investigator and research coordinator will review study materials for quality assurance periodically throughout the year. Protocol amendments will be submitted to the IRB and to NCCIH for prior approval before implementation. Protocol amendments will also be reported to the DSMB during scheduled meetings. Based on previous literature, we anticipate little to no study- 
related AEs except for some minor muscle soreness [42]. If minor soreness is reported, we will report the $\mathrm{AE}$ to the IRB and funder during our interim review, and we will discuss the event during our weekly meetings with the instructors and how to address this issue in order to minimize future AEs.

\section{Dissemination of findings}

The investigators will share research findings with the public and the scientific community through presentations at conferences and as requested by the NIH. Papers will be published in both peer-reviewed journals and reports to the NIH.

\section{Data sharing plan}

After the completion of the study, data will be made available to researchers upon review and approval of the submitted protocols by the research team. Researchers will need to sign an agreement to protect the confidentiality of the data and obtain prior approval from their respective IRBs, the Florida International University IRB, and the NIH. The full protocol is available via the principal investigator.

\section{Discussion}

The GEM Study is an exploratory clinical trial that will pilot test a TCQ intervention with older PLWH. If found acceptable to this population, TCQ programs could be an effective tool in managing both physical and psychological symptoms experienced by those living and aging with HIV. It will be an approach that can complement any medication regimen and is low impact enough that older PLWH could participate.

The study may pose potential limitations. One limitation may be the potential for diffusion of information. Participants recruited from similar sites may know one another and may share their experience during the trial period. To assist with minimizing this potential limitation, we will adjust for study site location. Another limitation may be generalizability as this trial is only among PLWH aged 50+ years of age. If the trial is found to be effective, it may not be generalizable to younger PLWH.

One of the strengths of the study is that the TCQ intervention will be compared to a similar control (SQG) condition to determine whether it is the physical aspect or the meditative aspect of qigong that is related to symptom management. Another unique aspect of this study is the home practice. Although the home practice has been included in previous studies using the intervention, this is the first time that we offer the video via streaming in addition to the DVD, and it is the first time that the video home practice will be developed in Spanish. For example, results may demonstrate whether older
PLWH will be amenable to seeing the TCQ home practice on their smartphones.

There is still much research to be done with qigong interventions. If found acceptable and feasible, a larger clinical trial will be proposed to further examine the efficacy of qigong for managing physical and psychological symptoms, as well as other aspects of disease progression such as immune functioning and cognitive impairment. In sum, qigong has the potential to cross racial, gender, and fitness levels; serves as a complementary activity to any HIV treatment regimen; and may be wellsuited for a population experiencing numerous physical and psychological symptoms such as older PLWH.

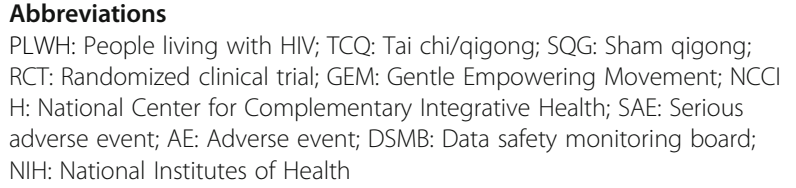

\section{Acknowledgements}

The authors would like to acknowledge Emil Varas for his contribution in preparing the manuscript for submission and Hsu Thadar and Victory Bryant for their contributions to the study.

\section{Authors' contributions}

Gl was the lead author on the manuscript. KF wrote and edited the data analysis plan. $\mathrm{LL}$ assisted in the description of the intervention. AA worked on the introduction and reviewing of the manuscript. CV and $\mathrm{NH}$ assisted in the study design and reviewing the manuscript and providing feedback. All authors have read an approved the manuscript.

\section{Funding}

This work was supported by the National Center for Complementary and Integrative Health (NCCIH) [R34 AT009966]. The content is solely the responsibility of the authors and does not necessarily represent the official views of the National Institutes of Health.

\section{Availability of data and materials}

Not applicable.

\section{Ethics approval and consent to participate}

This clinical trial was approved by the IRB at Florida International University and Arizona State University. Written consent will be received from all participants who choose to participate in this study. It is funded by the National Center for Complementary Integrative Health $(\mathrm{NCClH})$ and registered on www.clinicaltrials.gov [NCT03840525].

\section{Consent for publication}

N/A

\section{Competing interests}

No competing interests exist.

\footnotetext{
Author details

'Department of Epidemiology, Florida International University, 11200 SW 8th St AHC5, Miami, FL 33199, USA. ²Division of Natural Sciences, New College of Florida, 5800 Bay Shore Road, Sarasota, FL 34243, USA. ${ }^{3}$ College of Nursing and Health Innovation, Arizona State University, 550 N 3rd St, Phoenix, AZ 85004, USA. ${ }^{4}$ Department of Biostatistics, Florida International University, 11200 SW 8th St AHC5, Miami, FL 33199, USA. Division of Public Health, Family and Preventive Medicine, University of Utah School of Medicine, Salt Lake City, Utah 84132, USA. ${ }^{6}$ Division of Infectious Diseases and Global Public Health, University of California San Diego, La Jolla, CA 92093, USA.

${ }^{7}$ Department of Psychiatry, Semel Institute for Neuroscience and Human
} 
Behavior, The University of California, Los Angeles (UCLA), 760 Westwood Plaza, Los Angeles, CA 90095, USA.

\section{Received: 13 April 2020 Accepted: 8 September 2020}

Published online: 22 September 2020

\section{References}

1. Centers for Disease Control and Prevention. HIV Surveillance Report, 2017, vol. 29. Atlanta; 2018. Available at: https://www.cdc.gov/hiv/pdf/library/ reports/surveillance/cdc-hiv-surveillance-report-2017-vol-29.pdf.

2. Milanini B, Catella S, Perkovich B, et al. Psychiatric symptom burden in older people living with HIV with and without cognitive impairment: the UCSF HIV over 60 cohort study. AIDS Care. 2017;29(9):1178-85.

3. Brennan-Ing M, Karpiak SE, Seidel L. Health and psychosocial needs of LGBT older adults. Washington DC: Sage Publications; 2011. p. 19. Available at https://www.centeronhalsted.org/sage.pdf.

4. Sherr L, Clucas C, Harding R, et al. HIV and depression-a systematic review of interventions. Psychol Health Med. 2011;16(5):493-527.

5. Pereira M, Canavarro MC. Gender and age differences in quality of life and the impact of psychopathological symptoms among HIV-infected patients. AIDS Behav. 2011;15(8):1857-69.

6. Matthew Harris C, McKenzie R, Nayak S, et al. Graying of the HIV epidemic: a challenge for inpatient medicine providers. J Community Hosp Intern Med Perspect. 2015;5(6):29428

7. Mallon PW. Aging with HIV: osteoporosis and fractures. Curr Opin HIV AIDS. 2014;9(4):428-35.

8. Larkey L, Jahnke R, Etnier J, Gonzalez J. Meditative movement as a category of exercise: implications for research. J Phys Act Health. 2009:6(2):230-8.

9. Tsang HW, Cheung L, Lak DC. Qigong as a psychosocial intervention for depressed elderly with chronic physical illnesses. Int J Geriatr Psychiatry. 2002;17(12):1146-54

10. Abbott $\mathrm{R}$, Lavretsky $H$. Tai chi and Qigong for the treatment and prevention of mental disorders. Psychiatr Clin North Am. 2013;36(1):109.

11. Lee MS, Pittler MH, Ernst E. External qigong for pain conditions: a systematic review of randomized clinical trials. J Pain. 2007:8(11):827-31.

12. Guo X, Zhou B, Nishimura T, et al. Clinical effect of qigong practice on essential hypertension: a meta-analysis of randomized controlled trials. J Altern Complement Med. 2008;14(1):27-37.

13. Sancier KM. Therapeutic benefits of qigong exercises in combination with drugs. J Altern Complement Med. 1999;5(4):383-9.

14. Xin L, Miller YD, Brown WJ. A qualitative review of the role of qigong in the management of diabetes. J Altern Complement Med. 2007;13(4):427-34.

15. Oh B, Bae K, Lamoury G, Eade T, Boyle F, Corless B, Clarke S, Yeung A, Rosenthal D, Schapira L, Back M. The effects of tai chi and qigong on immune responses: a systematic review and meta-analysis. Medicines. 2020; 7(7):39.

16. Taylor-Piliae RE, Finley BA. Tai chi exercise for psychological well-being among adults with cardiovascular disease: a systematic review and metaanalysis. Eur J Cardiovasc Nurs. 2020:1-12. Available from https://journals. sagepub.com/doi/pdf/10.1177/1474515120926068?casa_token=peh_ZtMe22 YAAAAA:xBB10xa9W2XynspuWR6q9wcLvT2hbzOhw4obSDh xe7UFmR9Pna8v_2VbSVGiC7RRTbr7SZMCtfTt_A. https://doi.org/10.1177/ 1474515120926068.

17. Liu F, Cui J, Liu X, Chen KW, Chen X, Li R. The effect of tai chi and gigong exercise on depression and anxiety of individuals with substance use disorders: a systematic review and meta-analysis. BMC Complement Med Ther. 2020;20:1-1.

18. Guan $Y$, Hao $Y$, Guan $Y$, Wang H. Effects of tai chi on essential hypertension and related risk factors: a meta-analysis of randomized controlled trials. Rehabil Med. 2020;52(5):1-2.

19. Kojima G. Frailty as a predictor of disabilities among community-dwelling older people: a systematic review and meta-analysis. Disabil Rehabil. 2017; 39(19):1897-908.

20. Galantino ML, Shepard K, Krafft L, et al. The effect of group aerobic exercise and t'ai chi on functional outcomes and quality of life for persons living with acquired immunodeficiency syndrome. J Altern Complement Med. 2005;11(6):1085-92.

21. McCain NL, Gray DP, Elswick RK Jr, et al. A randomized clinical trial of alternative stress management interventions in persons with HIV infection. J Consult Clin Psychol. 2008;76(3):431.
22. Robins JL, McCain NL, Gray DP, et al. Research on psychoneuroimmunology: tai chi as a stress management approach for individuals with HIV disease. Appl Nurs Res. 2006;19(1):2-9.

23. Spirduso WW, Poon LW, Chodzko-Zajko WJ. Using resources and reserves in an exercise-cognition model. In: Spirduso WW, Poon LW, Chodzko-Zajko WJ, editors. Exercise and its mediating effects on cognition. Champaign: Human Kinetics; 2008. p. 3-28.

24. Dennis ML, Titus JC, White MK, et al. Global appraisal of individual needs: administration guide for the GAIN and related measures. Bloomington: Chestnut Health Systems; 2003.

25. Jahnke RA, Larkey LK, Rogers C. Dissemination and benefits of a replicable Tai Chi and Qigong program for older adults. Geriatr Nurs. 2010;31(4):27280 .

26. Kalichman SC, Simbayi LC, Cloete A, et al. Measuring AIDS stigmas in people living with HIV/AIDS: the Internalized AIDS-Related Stigma Scale. AIDS Care. 2009;21(1):87-93.

27. Sarason IG, Sarason BR, Shearin EN, Pierce GR. A brief measure of social support: practical and theoretical implications. J Soc Pers Relat. 1987;4(4): 497-510.

28. Skinner HA. The drug abuse screening test. Addict Behav. 1982;7:363-71.

29. Bush K, Kivlahan DR, MCDonell MB, Fihn SD, Bradley KA. The AUDIT alcohol consumption questions (AUDIT-C): an effective brief screening test for problem drinking. Arch Intern Med. 1998;158:1789-95.

30. Parsons JT, Starks TJ, Millar BM, Boonrai K, Marcotte D. Pattersn of substance use among HIV-positive adults over 50: implications for treatment and medication adherence. Drug Alcohol Depend. 2014;139:33-40.

31. Cole JC, Rabin AS, Smith TL, Kaufman AS. Development and validation of a Rasch-derived CES-D short form. Psychol Assess. 2004;16(4):360.

32. Ng F, Trauer T, Dodd S, et al. The validity of the 21-item version of the Depression Anxiety Stress Scales as a routine clinical outcome measure. Acta Neuropsychiatr. 2007;19(5):304-10.

33. Holzemer WL, Hudson A, Kirksey KM, et al. The revised sign and symptom check-list for HIV (SSC-HIVrev). J Assoc Nurses AIDS Care. 2001;12(5):60-70.

34. Belza BL, Henke CJ, Yelin EH, et al. Correlates of fatigue in older adults with rheumatoid arthritis. Nurs Res. 1993;42(2):93-9.

35. Morisky DE, Ang A, Krousel-Wood M, Ward HJ. Predictive validity of a medication adherence measure in an outpatient setting. J Clin Hypertens. 2008;10(5):348-54

36. Larkey L, Szalacha L, Rogers $C$, et al. Measurement pilot study of the Meditative Movement Inventory (MMI). J Nurs Meas. 2012;20(3):230-43.

37. Marin G, Marin BV. Applied social research methods series, Vol. 23. Research with Hispanic populations. Thousand Oaks: Sage Publications, Inc; 1991.

38. Sekhon M, Cartwright M, Francis JJ. Acceptability of healthcare interventions: an overview of reviews and development of a theoretical framework. BMC Health Serv Res. 2017;17(1):88.

39. Bowen DJ, Kreuter M, Spring B, et al. How we design feasibility studies. Am J Prev Med. 2009;36(5):452-7.

40. Lancaster GA, Dodd S, Williamson PR. Design and analysis of pilot studies: recommendations for good practice. J Eval Clin Pract. 2004;10(2):307-12.

41. Newcombe RG. Two-sided confidence intervals for the single proportion: comparison of seven methods. Stat Med. 1998;17(8):857-72.

42. Wayne PM, Berkowitz DL, Litrownik DE, Buring JE, Yeh GY. What do we really know about the safety of tai chi?: a systematic review of adverse event reports in randomized trials. Arch Phys Med Rehabil. 2014;95(12): 2470-83.

\section{Publisher's Note}

Springer Nature remains neutral with regard to jurisdictional claims in published maps and institutional affiliations. 